\title{
Measurement of Stone Diameter with Three Sizes of Automatically Fixating Stone Baskets"
}

\author{
Jens Cordes $^{1 \#}$, Felix Nguyen ${ }^{1}$, Wolfgang Pinkowski ${ }^{2}$, Dieter Jocham ${ }^{1}$ \\ ${ }^{1}$ Urological Clinic and Policlinic of the University of Luebeck, University Medical Center Schleswig-Holstein (UKSH), Lübeck \\ Germany \\ ${ }^{2}$ Urotech GmbH, Achenmühle, Germany \\ Email: "Jens.Cordes@uksh.de
}

Received February 5, 2013; revised March 6, 2013; accepted March 14, 2013

Copyright (C) 2013 Jens Cordes et al. This is an open access article distributed under the Creative Commons Attribution License, which permits unrestricted use, distribution, and reproduction in any medium, provided the original work is properly cited.

\begin{abstract}
Background: Complications after endoscopic retrieval of kidney and ureter stones are obviously related to the size of the stones as well as the experience of the surgeon and other factors. During the procedure it is sometimes difficult for surgeons to estimate stone size and therefore give prognostic advises. The visual perception of the stone size depends on the shape, colour, distance to the renoscope and dilatation of the ureter. This is the so-called binding problem, because shape, color and direction of motion are processed separately by different population of optical neurons. In order to establish a better prognostic rational especially for less experienced surgeons, we established an intra operative semi-quantitative measurement of the stone size supported by a stone basket. Materials and Methods: We modified the tipped, nitinol stone baskets from the company Urotech with diameters of 2.5, 3.0 and 4 FR. The handle of this basket has a spring mechanism, which automatically closes the basket and provides a predefined fixation force of the stones within the basket. On the handle we established a non-linear scale in $\mathrm{mm}$ by grabbing standardized balls or standardized screws. Result: The scales are nonlinear because of the nonlinear relation between the diameter of the stone and the distance of the slider. Also the scales differ in between the basket size, because of the different strain conditions due to the different wire sizes and materials or the spring and basket. Conclusion: This scale could be an important orientation for a surgeon during endourological procedures to estimate stone sizes. After further clinical experience a semi-quantitative visualization like green, yellow and red colors could help to predict potential complications due to large stone sizes. Finally it could bevery interesting for other disciplines like gastroenterology.
\end{abstract}

Keywords: Stone Basket; Automatically Fixating Stone Basket; Measurement of Stone Size

\section{Introduction}

An increase in the prevalence of urolithiasis to $5 \%$, i.e. an increase of 25 percent within 20 years, was ascertained in Germany in 2000 [1].

The further development of instrumental technique towards extremely thin and even flexible ureteroscopes as well as modern lithotripsy procedures with various energy sources has once again placed special emphasis on endoscopic and percutaneous minimally invasive techniques [2]. Procedures increasingly carried out in Germany are endourological lithotripsy of ureteral and kid-

\footnotetext{
"Competing interests: The authors declare that they have no competing interests.

Authors' contributions: WP gave technical support and the baskets. JC and FN carried out the studies, JC drafted the manuscript. All authors read and approved the final manuscript.

${ }^{\#}$ Corresponding author.
}

ney stones. Superelastic baskets are often used to extract the stone or to stabilize the stone during the intervention [3]. But there are still complications based on different reasons. One important factor for adverse events is the experience of the surgeons with this challenging technique.

Elashry, O.M. et al. 2008 showed in a 15-year singlecentre experience of ureteroscopic management of lower ureteric calculi a decrease in ureteric perforation from $3.3 \%$ to $0.5 \%$, in ureteric avulsion from $1.3 \%$ to $0.1 \%$ and in ureteric stricture from $0.7 \%$ to $0.1 \%$ [4].

A positive history of ureteral surgery, stones $>5 \mathrm{~mm}$ in width, a dilated proximal ureter, kidneys that failed to excrete contrast medium, stones above the ischial spines, and involvement of junior urologist were factors that were associated with a statistically significant higher incidence of intra operative complications [5]. 
Sometimes it is difficult for surgeons to estimate the widths or diameter of the stone. The perception depends on the shape, colour, distance to the renoskope and dilatation of the ureter. This is the so-called binding problem [6], because shape, color and direction of motion are processed separately by different population of neurons [7].

In order to help to estimate the stone size and therefore to potentially predict post operative adverse events we implement an intra-operative, quantitative stone measurement modifying an existing stone basket.

\section{Material and Methods}

We used nitinol standard stone baskets from the company Urotech with diameters of 2.5, 3.0, and 4.0 FR.

The handle was developed by Urotech together with Prof. S. Lahme (Pforzheim, Germany).

This handle is characterized by several unique design elements. It has an automatic fixation of the stone in the basket with a spring mechanism and a dis- and reconnectable handle so that the endoscope can be removed while the retrieval basket is situated and the handle afterwards can be reconnected again.

On the handle we established a non-linear scale in $\mathrm{mm}$ by grabbing standardized balls, normally used for ball bearings or standardized screws. The standard called "DIN: Deutsche Industrie Norm" for the balls and was DIN ISO 281.

The grabbing force was provided by the spring mechanism of the handgrip Figure 1.

For the $2 \mathrm{~mm}$ diameter measurements with the 2.5 and 3.0 Ch. baskets we had to use cylindrical steel rods, because these two baskets could not grip balls with the diameter of $2 \mathrm{~mm}$. Smaller diameters than $2 \mathrm{~mm}$ were not measurable.

We also tried to establish a scale on a handle of the 1.2 FR and 1.8 FR tipless baskets in order to establish this measurement also for kidney stones.

Unfortunately this was not possible because of the strong forces provided by the spring and the weak, thin nitinol basket. After gripping a ball or a steel rod the handle always went into the same position.

\section{Results}

Figure 1 shows the final handle with the scale. The scale starts at $2 \mathrm{~mm}$, because smaller balls or steel rods couldn't be differentiated on the grip. For the small diameter of $2 \mathrm{~mm}$ we had to use cylindrical steel rods for the 2.5 and the $3.0 \mathrm{Ch}$. baskets. These two baskets couldn't grip balls of this small size, because of the helical shape of these baskets.

The scale is nonlinear because of the nonlinear relation between the diameter of the stone and the distance of the slider and also because of the different materials of the basket (nitinol) and the spring (stainless steel).

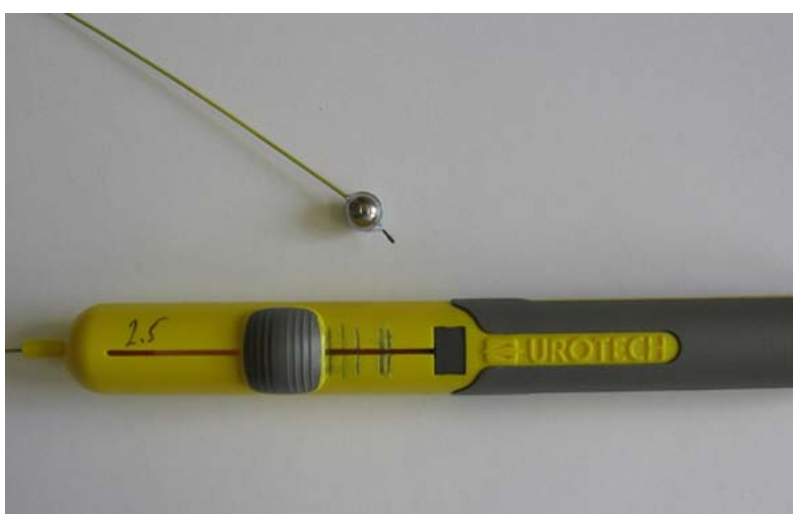

Figure 1. On the handle we established a non-linear scale in mm by grabbing standardized balls, normally used for ball bearings and standardized cylindrical steel rods for the smaller size.

The reason for this can be find in the different strain conditions due to the difference in relation of stress and strain of steel and nitinol (Figure 2).

While steel shows an increase in stress in the beginning nitinol shows an increase in strain. The scales also differ with the size of the basket (Figure 3) because we used the same handle with the same spring force with different diameters of the basket wires, so that thinner nitinol is more deformed. Furthermore the closed position of the slider is located in different positions due to different basket sizes.

\section{Discussion}

Under experimental conditions and under consideration of the used materials it is possible to measure stone diameters with a stone retrieval basket. With this information it might be possible to increase the safety and effectiveness of endourological procedures because the surgeon is able to estimate the post operative prognosis based on his own or known rational between stone size and potential complications [7].

Based on this additional information during the procedure, we think that our scale could contribute to increase the safety of endourological procedures, especially for less experienced surgeons. Our proposal would be that the scale has also a colored background beside the numbers as shown in Figure 3 in order to have a brief semi-quantitative measurement of the stone size. Based on the above mentioned rational small stone sizes less than $5 \mathrm{~mm}$ could marked with a green colour, medium stone sizes between 5 and $8 \mathrm{~mm}$ could have a yellow colour and stone over $8 \mathrm{~mm}$ could be marked with a red colour (Figure 3).

The green area is based on the study of Abdelrahim A.F. et al. 2008 [5] in which stones $>5 \mathrm{~mm}$ in width were associated with a statistically significant higher incidence of intra operative complications. The yellow area is just a 


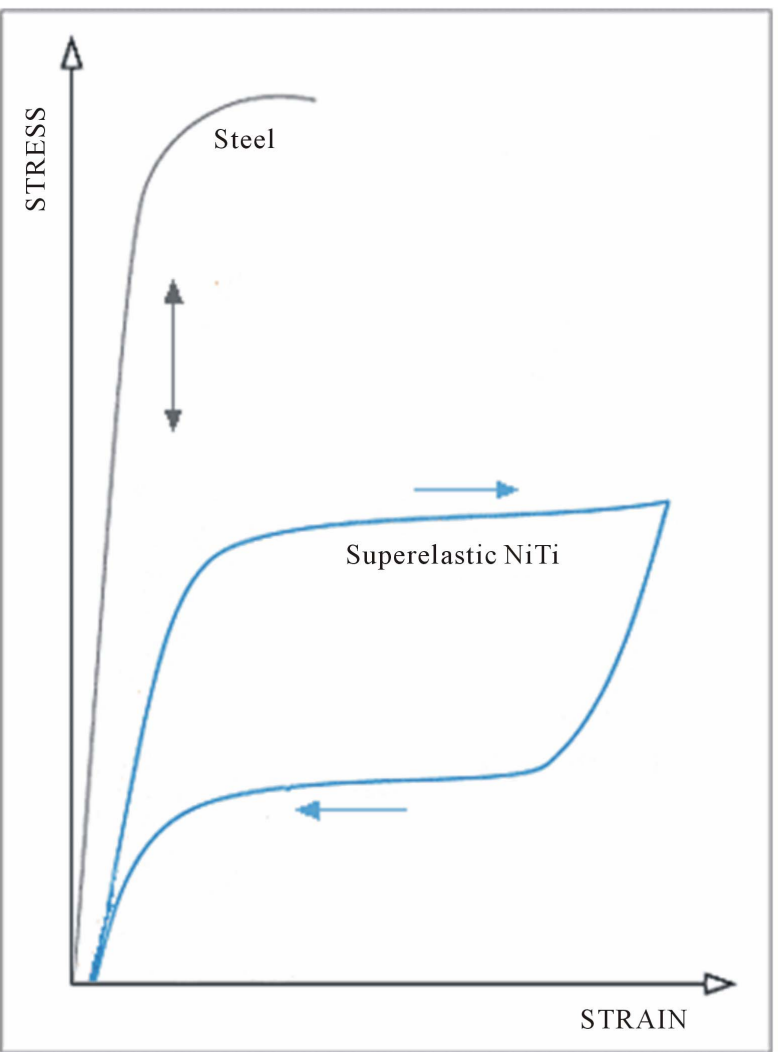

Figure 2. stress and strain curve of nitinol and stainless steel: At the beginning steel is not flexible and shows increasing stress whereas nitinol shows a strain in the beginning and only a moderate in stress $[8]$.
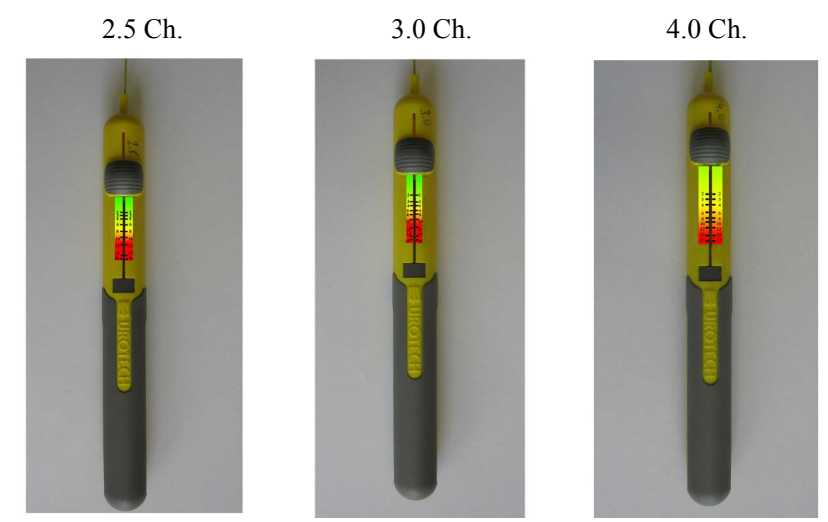

Figure 3. Proposal for a definitive scale for these tree different baskets $(2.5,3.0$ and $4.0 \mathrm{Ch}$.). The nonlinear scale differs because we use the same handle with different baskets and the slider at the handle differs in closed position due to different basket sizes.

proposal and should be further investigated. In the red area the operating surgeon should manipulate very careful.

Stone size is usually measured on radiological imaging (Non Contrast CT or intravenous Pyelography). It is not possible to measure a stone volume in an intravenous
Pyelography or intra-operative, so it could be important also from the forensic point of view to measure a stone size before destroying a stone in the ureter. Also it could be a useful tool in combination with a flexible ureterrenoscopy (URS), whether the stone should be disintergrated before extraction. In a flexible URS the estimation of the stone size is more difficult because of impaired vision. Until now we couldn't establish a scale on tipless baskets but maybe some improvements of the basket handle like changing the material of the spring could solve this limitation.

The alloy of Nickel and Titanium is elastic so that the force of the grab should be done with a standard power as our grip dose with the spring. This flexibility is not linear as the flexibility of steel resulting in the non-linear scale (Figure 2).

However, ureteral stones have not the shape of ball bearings or cylindrical rods but there is no other useful form for a standard.

Until today the scale is only developed using nonclinical, experimental methods, which currently limits the conclusion of this study and further clinical investigations based on new and modified baskets with this automatically fixation handle are required to prove this concept.

\section{Conclusion}

We think that our scale could be a contribution and important orientation for a surgeon conducting endourological stone retrievals to increase safety and effectiveness of the procedure and further clinical investigations based on new and modified baskets with this automatically fixation handle required to prove this concept. Finally it could be very interesting for other disciplines like gastroenterology.

\section{REFERENCES}

[1] A. Hesse, E. Brändle, D. Wilbert, K.-U. Köhrmann and P. Alken, "Study on the Prevalence and Incidence of Urolithiasis in Germany Comparing the Years 1979 vs. 2000," European Urology, Vol. 44, No. 6, 2003, pp. 709713. doi:10.1016/S0302-2838(03)00415-9

[2] S. C. Müller, R. Hofmann, K.-U. Köhrmann and A. Hesse, "Epidemiologie, Instrumentelle Therapie und Metaphylaxe des Harnsteinleidens," Deutsches Ärzteblatt, Vol. 101, No. 19, 2004, pp. 1331-1336.

[3] D. Stoeckel, "Nitinol Medical Devices and Implants," Minimal Invasive Therapy \& Allied Technology, Vol. 9, No. 2, 2000, pp. 81-88. doi:10.3109/13645700009063054

[4] O. M. Elashry, A. K. Elgasmasy, M. A. Sabaa, M. AboElenien, M. A. Omar, H. H. Eltatawy and S. A. El-Abd, "Ureteroscopic Management of Lower Ureteric Calculi: A 15-Year Single-Centre Experience," British Journal of Urology International, Vol. 102, No. 8, 2008, pp. 1010- 
1017. doi:10.1111/j.1464-410X.2008.07747.x

[5] A. F. Abdelrahim, A. Abdelmaguid, H. Abuzeid, M. Amin, El S. Mousa and F. Abdelrahim, "Rigid Ureteroscopy for Ureteral Stones: Factors Associated with Intraoperative Adverse Events," Journal of Endourology, Vol. 22, No. 2, 2008, pp. 277-280.

doi:10.1089/end.2007.0072

[6] A. Treisman, "The Binding Problem," Current Opinion
Neurobiology, Vol. 6, No. 2, 1996, pp. 171-178. doi:10.1016/S0959-4388(96)80070-5

[7] M. Masahiko, M. Shigemitsu and M. Hiromi, "Attribute Pair-Based Visual Recognition and Memory," PLoS One, Vol. 5, No. 5, 2010, p. e9571. doi:10.1371/journal.pone.0009571

[8] D. Stoeckel, "Umformung von NiTi-Legierungen-Einen Herausforderung," NDC, 2001, pp. 141-157. 